International Journal of Pure and Applied Mathematics

Volume 103 No. 2 2015, 169-185

ISSN: 1311-8080 (printed version); ISSN: 1314-3395 (on-line version)

url: http://www.ijpam.eu

doi: http://dx.doi.org/10.12732/ijpam.v103i2.4

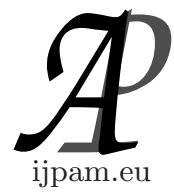

\title{
TRANSLATES OF VAGUE NORMAL GROUPS
}

\author{
B. Nageswararao ${ }^{1 \S}$, N. Ramakrishna ${ }^{2}$, T. Eswarlal ${ }^{3}$ \\ ${ }^{1}$ Department of Mathematics \\ KL University \\ Vaddeswaram, 522 502, Guntur Dist. Andhra Pradesh, INDIA \\ ${ }^{2}$ Department of Mathematics \\ Mrs. A.V.N. College \\ Visakhapatnam, Andhra Pradesh, INDIA \\ ${ }^{3}$ Department of Mathematics \\ KL University \\ Vaddeswaram, 522 502, Guntur Dist. Andhra Pradesh, INDIA
}

\begin{abstract}
In this paper we introduce translate operators on vague cut set, vague normal groups, and studied their properties. These concepts are used in the development of some important results and theorems about translates of vague homomorphism, vague normal groups. Also some of their important properties have been investigated.
\end{abstract}

AMS Subject Classification: 08A72, 20N25, 03E72

Key Words: vague set, vague group, vague normalizer, vague centralizer, translates of vague group, translate of vague $A_{(\alpha, \beta)}$ cut set, translates of Vague normal group

\section{Introduction}

The concept proposed by L.A. Zadeh [14] defining a fuzzy subset $A$ of a given universe $\mathrm{X}$ characterizing the membership of an element $x$ of $X$ belonging to $A$

Received: January 29, 2015

(C) 2015 Academic Publications, Ltd.

$\S$ Correspondence author url: www.acadpubl.eu 
by means of a membership function $\mu_{A}$ defined from $X$ into $[0,1]$ has revolutionized the theory of Mathematical modeling decision making etc. In handling the imprecise real life situations mathematically. Now several branches of fuzzy mathematics like fuzzy algebra, fuzzy topology, fuzzy control theory, fuzzy measure theory etc. have emerged. But in the decision making, the fuzzy theory takes care of membership of an element $\mathrm{x}$ only, that is the evidence against $\mathrm{x}$ belonging to $\mathrm{A}$. It is felt by several decision makers and researchers that in proper decision making, the evidence belongs to A and evidence not belongs to $\mathrm{A}$ are both necessary and how much $\mathrm{X}$ belongs to $\mathrm{A}$ or how much $\mathrm{x}$ does not belongs to A are necessary.

Several generalizations of Zadeh. L. A'sfuzzy set theory have been proposed, such as L-fuzzy sets (see [4]), Interval valued fuzzy sets. Intuitionistic fuzzy sets by K.T. Atanassov [1] and W.L. Gahu, D.J. Buehrer Vague sets, see [3], are mathematically equivalent. Any such set $\mathrm{A}$ of a given Universe $\mathrm{X}$ can be characterized by means of a pair of function $\left(t_{A}, f_{A}\right)$ where $t_{A}$ and $f_{A}$ are function from $\mathrm{X}$ in to [01] such that $0 \leq t_{A}(x)+f_{A}(x) \leq 1$ for all $\mathrm{x}$ in $\mathrm{X}$. The set $t_{A}$ is called the truth function and the set $f_{A}$ is called false function or non membership function and $t_{A}(x)$ gives the evidence of how much $\mathrm{x} \in \mathrm{A} f_{A}(x)$ gives the evidence of how much $\mathrm{x}$ does not $\in \mathrm{A}$. These concepts are being applied in several areas like decisionmaking, fuzy control, knowledge discovery and fault diagonsis etc. I t is belived the vague sets (or equivalently instuitionisticfuzzy sets) will more useful in decision making, and other areas of Mathematical modeling. Through K.T. Atanassov [1] instuitionistic fuzzysets, W.L. Gau and D.J. Buehrer [3] and some other areas of Mathematical modeling. Since then the theory fuzzy sets developed extensivelyand embraced almost all subjects like engineering science and Technology. But the membership function $\mu_{A}$ gives only a approximation belong to A. To avid this and obtain a better estimation and analysis of data decision making.

W.L. Gau and D.J. Bueher in [3] have initiated the study of vague sets with the hope that they form a better tool to understand, interpret and solve real life problems which are in general vague, than the theory of Fuzzy sets do. Ranjit Biswas [9] initiated the study of vague groups and N. Ramakrishna [8], [10], [11], T. Eswarlal [2], [8], [10] create extended the study of vague algebra. The objective of this paper is to contribute further to the study of vague algebra by introducing concepts of translate operators on vague cut set, translate operators on vague homomorphism and translate operators on vague normal groups. 


\section{Preliminaries}

We give here a review of some definitions and results which one may find in W.L. Gau and D.J. Buehrer [3], Ranjit Biswas [9], N. Ramakrishna [8], [10], [11].

Definition 1.1. A vague set $A$ in the universe of discourse $U$ is a pair $\left(t_{A}, f_{A}\right)$ where $t_{A}: U \rightarrow[0,1], f_{A}: U \rightarrow[0,1]$, are mappings such that $t_{A}(u)+$ $f_{A}(u) \leq 1$, for all $u \in U$. The functions $t_{A}$ and $f_{A}$ are called true membership function and false membership function respectively.

Definition 1.2. The interval $\left[t_{A}(u), 1-f_{A}(u)\right]$ is called the vague value of $\mathrm{u}$ in $A$, and it is denoted by $V_{A}(u)$, i.e. $V_{A}(u)=\left[t_{A}(u), 1-f_{A}(u)\right]$.

Definition 1.3. Let $(G, *)$ be a group. A vague set $A$ of $G$ is called a vague group of $G$ if, for all $\mathrm{x}, \mathrm{y}$ in $G, V_{A}(x y) \geq \min \left\{V_{A}(x), V_{A}(y)\right\}$ and $V_{A}\left(x^{-1}\right) \geq V_{A}(x)$, i.e.

$$
t_{A}(x y) \geq \min \left\{t_{A}(x), t_{A}(y)\right\}, \quad f_{A}(x y) \leq \max \left\{f_{A}(x), f_{A}(y)\right\}
$$

and

$$
t_{A}\left(x^{-1}\right) \geq t_{A}(x), \quad f_{A}\left(x^{-1}\right) \leq f_{A}(x) .
$$

Here the element $x y$ stands for $x * y$.

Definition 1.4. Let $A$ be a vague set of a universe $G$ with true-membership function $t_{A}$, and false membership function $f_{A}$. For $\alpha, \beta \epsilon[0,1]$ with $\alpha \leq \beta$, the $(\alpha, \beta)$ cut or vague cut of a vague set $A$ is the crisp subset of $G$ is given by $A_{(\alpha, \beta)}=\left\{x \mid x \epsilon G, V_{A}(x) \geq[\alpha, \beta]\right\}$, i.e. $A_{(\alpha, \beta)}=\left\{x \mid x \epsilon G, t_{A}(x) \geq \alpha\right.$, and $\left.1-f_{A}(x) \geq \beta\right\}$.

Definition 1.5. The $\alpha$-cut, $A_{\alpha}$ of the vague set $A$ is the $(\alpha, \alpha)$ cut of $A$, and hence given by $A_{\alpha}=\left\{x \mid x \in G, t_{A}(x) \geq \alpha\right\}$.

Definition 1.6. Let $A$ be a Vague group of a group $G$ Then $A$ is Called Vague normal group if for all $\mathrm{x}, \mathrm{y} \in G, V_{A}(x y)=V_{A}(y x)$. Alternatively, we can say that, a vague $\operatorname{group} A$ is said to be vague normal group of $G$ if $V_{A}(x)=V_{A}\left(y x y^{-1}\right)$ for all $\mathrm{x}, \mathrm{y} \in G$.

We now introduce the following definitins. 
Definition 1.7. Let $A$ be a Vague group of a group $G$ then the set $\mathbb{N}(A)=\left\{a \in G \mid V_{A}\left(a x a^{-1}\right)=V_{A}(x)\right.$ for all $\left.a, \in G\right\}$ is called vague normalizer of $A$.

Definition 1.8. Let $A$ be a vague group of a group $G$ Then the set

$$
\mathbb{C}(A)=\left\{a \in G \mid V_{A}([a, x])=V_{A}(e) \text { for all } x \in G\right\}
$$

is called vague centralizer of $A$, where $[\mathrm{a}, \mathrm{x}]=a^{-1} x^{-1} a x$.

Defination 1.9. A function $f: G \rightarrow G^{1}$ is said to be vague homomorphism if $f(a . b)=f(a) . f(b)$ for all $a, b \in G$.

\section{Translate Operators on Vague Cut-Set}

Definition 2.1. Let $\mathrm{A}$ be a vague set of universal set $\mathrm{X}$ then $A_{(\alpha, \beta)}$ cut-set of a vague translation operators can be defined by:

(a) $\left.T_{\theta+}\left(A_{(} \alpha \beta\right)\right)=\left(t_{A_{(\alpha, \beta) T_{\theta+}}}(x), f_{A_{(\alpha, \beta) T_{\theta+}}}(x)\right)$, where $t_{A_{(\alpha, \beta) T_{\theta+}}}(x)=\min \left\{t_{A_{(\alpha, \beta)}}(x)+\theta, 1\right\}$ and $f_{A_{(\alpha, \beta) T_{\theta+}}}(x)=\max \left\{f_{A_{(\alpha, \beta)}}(x)-\theta, 0\right\}$.

(b) $\left.T_{\theta-}\left(A_{(} \alpha \beta\right)\right)=\left(t_{A_{(\alpha, \beta) T_{\theta-}}}(x), f_{A_{(\alpha, \beta) T_{\theta-}}}(x)\right)$, where $t_{A_{(\alpha, \beta) T_{\theta-}}}(x)=\max \left\{t_{\left.A_{(\alpha, \beta}\right)}(x)-\theta, 0\right\}$ and $f_{A_{(\alpha, \beta) T_{\theta-}}}(x)=\min \left\{f_{A_{(\alpha, \beta)}}(x)+\theta, 1\right\}$.

Here: $A_{(\alpha, \beta)}=\left\{x \in X, t_{A}(x) \geq \alpha, f_{A}(x) \leq \beta\right\}$, where $\theta$, $\alpha$, and $\beta \in[0,1]$; $\left.T_{\theta+}\left(A_{(} \alpha \beta\right)\right)$ and $\left.T_{\theta-}\left(A_{(} \alpha \beta\right)\right)$ are called translation of vague cut-set increasing operator and translation of vague cut-set decreasing operator respectively.

Remark 2.2. Let $\mathrm{A}$ be a vague set of a universal set $\mathrm{X}, A_{(\alpha, \beta)}$ be a vague cut-set then

$$
0 \leq t_{A_{(\alpha, \beta) T_{\theta+}}}(x)+f_{A_{(\alpha, \beta) T_{\theta+}}}(x) \leq 1 .
$$

Example 2.3. Let $X=\{1,-1\}, A=\{(1,0.6,0.02),(-1,0.5,0.04)\}$ and $\alpha=0.4, \beta=0.1$ and $\theta=0.2$.

Let $X=\{1,-1\}, A=\{(1,0.6,0.02),(-1,0.5,0.04)\}$ and $\alpha=0.4, \beta=0.1$ and $\theta=0.2$ since $A_{(\alpha, \beta)}=\left\{x \in X, t_{A}(x) \geq \alpha, f_{A}(x) \leq \beta\right\}$. 
Now $\left.T_{\theta+}\left(A_{(} \alpha, \beta\right)\right)=\left(t_{A_{(\alpha, \beta) T_{\theta+}}}(x), f_{A_{(\alpha, \beta) T_{\theta+}}}(x)\right)$, where $t_{A_{(\alpha, \beta) T_{\theta+}}}(x)=$ $\min \left\{t_{\left.A_{(\alpha, \beta}\right)}(x)+\theta, 1\right\}$ and

$$
\begin{gathered}
f_{A_{(\alpha, \beta) T} T_{\theta+}}(x)=\max \left\{f_{\left.A_{(\alpha, \beta}\right)}(x)-\theta, 0\right\} \\
t_{A_{(\alpha, \beta) T_{\theta+}}}(1)=\min \left\{t_{\left.A_{(\alpha, \beta}\right)}(1)+\theta, 1\right\}=\min \{0.6+0.2,1\}=0.8 \\
f_{A_{(\alpha, \beta) T_{\theta+}}}(1)=\max \left\{f_{A_{(\alpha, \beta)}}(1)-\theta, 0\right\}=\max \{0.02-0.2,0\}=0 \\
\Rightarrow t_{A_{(\alpha, \beta) T} T_{\theta+}}(1)+f_{A_{(\alpha, \beta) T_{\theta+}}}(1)=0.8+0=0.8 \leq 1 .
\end{gathered}
$$

Therefore $\left.t_{A_{(\alpha, \beta) T}{ }_{\theta+}}(1)+f_{A_{(\alpha, \beta) T_{\theta+}}}(1) \in T_{\theta+}\left(A_{(} \alpha \beta\right)\right)$,

$$
\begin{gathered}
t_{A_{(\alpha, \beta) T_{\theta+}}}(-1)=\min \{0.5+0.2,1\}=\min \{0.7,1\}=0.7, \\
f_{A_{(\alpha, \beta) T_{\theta+}}}(-1)=\max \{0.04-0.2,0\}=\max \{-0.16,0\}=0, \\
\left.\Rightarrow t_{A_{(\alpha, \beta) T_{\theta+}}}(-1)+f_{A_{(\alpha, \beta) T_{\theta+}}}(-1)=0.7+0=0.7 \in T_{\theta+}\left(A_{(} \alpha \beta\right)\right) .
\end{gathered}
$$

Therefore $0 \leq t_{A_{(\alpha, \beta) T_{\theta+}}}(-1)+f_{A_{(\alpha, \beta) T_{\theta+}}}(-1) \in T_{\theta+}\left(A_{(\alpha, \beta)}\right.$. Hence $T_{\theta+}\left(A_{(\alpha, \beta)}\right.$ is a translate of vague $A_{(\alpha, \beta)}$ cut-set. similarly we have to show that $T_{\theta-A_{(\alpha, \beta)}}$ is a translate of vague $A_{(\alpha, \beta)}$ cut-set.

Example 2.4. Let $X=\left\{1, w, w^{2}\right\}, A=\left\{(1,0.3,0.6),(w, 0.1,0.25),\left(w^{2}\right.\right.$, $0.5,0.1)\}$.

It is given: $\mathrm{X}=\left\{1, w, w^{2}\right\}$ and $A=\left\{(1,0.3,0.1),(w, 0.1,0.25),\left(w^{2}, 0.5,0.1\right)\right\}$ $\alpha=0.3, \beta=0.2, \theta=0.4$. Since $A_{(\alpha, \beta)}=\left\{x \in X: t_{A}(x) \geq \alpha, 1-f_{A}(x) \geq \beta\right\}$, where $t_{A_{(\alpha, \beta) T_{\theta-}}}(x)=\max \left\{t_{A_{(\alpha, \beta)}}(x)-\theta, 0\right\}$ and

$$
\begin{aligned}
& f_{A_{(\alpha, \beta) T_{\theta-}}}(x)=\min \left\{f_{\left.A_{(\alpha, \beta}\right)}(x)+\theta, 1\right\}, \\
& t_{A_{(\alpha, \beta) T_{\theta}}}(1)=\max \left\{t_{\left.A_{(\alpha, \beta}\right)}(1)-\theta, 0\right\}=\max \{0.3-0.4,1\}=1, \\
& f_{A_{(\alpha, \beta) T_{\theta-}}}(1)=\min \left\{f_{\left.A_{(\alpha, \beta}\right)}(1)-\theta, 0\right\}=\max \{0.1-0.4,0\}=0, \\
& \Rightarrow t_{A_{(\alpha, \beta) T_{\theta-}}(1)+f_{A_{(\alpha, \beta) T_{\theta-}}}(1)}=1+0=1 \leq 1 .
\end{aligned}
$$

Therefore $\left.t_{A_{(\alpha, \beta) T_{\theta-}}}(1)+f_{A_{(\alpha, \beta) T_{\theta-}}}(1) \in T_{\theta-}\left(A_{(} \alpha \beta\right)\right), t_{A_{(\alpha, \beta) T_{\theta-}}}(w)=\max \{0.1-$ $0.4,1\}=\min \{-0.3,1\}=1, f_{A_{(\alpha, \beta) T_{\theta-}}}(w)=\min \{0.25-0.4,0\}=\max \{-0.15,0\}=$ $0, \Rightarrow t_{A_{(\alpha, \beta) T_{\theta-}}}(w)+f_{A_{(\alpha, \beta) T_{\theta-}}}(w)=1+0=1 \in T_{\theta-}\left(A_{(} \alpha \beta\right)(w), t_{A_{(\alpha, \beta) T_{\theta-}}}\left(w^{2}\right)=$ $\max \{0.5-0.4,1\}=\min \{0.1,1\}=0.1, f_{A_{(\alpha, \beta) T_{\theta-}}}\left(w^{2}\right)=\min \{0.1-0.4,0\}=$ $\max \{-0.3,0\}=0 \Rightarrow t_{A_{(\alpha, \beta) T_{\theta-}}}\left(w^{2}\right)+f_{A_{(\alpha, \beta) T_{\theta-}}}\left(w^{2}\right)=0.1+0=0.1 \in T_{\theta-}\left(A_{(\alpha, \beta)}\right)$. 
Hence $T_{\theta-}\left(A_{(\alpha, \beta)}\right.$ is a translate of vague $A_{(\alpha, \beta)}$ cut-set. Similarly we have to show that $T_{\theta+A_{(\alpha, \beta)}}$ is a translate of vague $A_{(\alpha, \beta)}$ cut-set.

Theorem 2.5. If $P$ and $Q$ be two vague sets of a universal set $X$, then following holds:

(1) $A_{(\alpha, \beta)}(P) \subseteq A_{(\gamma, \delta)}(Q)$ if $\alpha \geq \gamma, \beta \leq \delta$,

(2) $A_{(1-\beta, \beta)}(P) \subseteq A_{(\alpha, \beta)}(P) \subseteq A_{(\alpha, 1-\alpha)}(P)$,

(3) $P \subseteq Q \Rightarrow A_{(\alpha, \beta)}(P) \subseteq A_{(\alpha, \beta)}(Q)$,

(4) $A_{(\alpha, \beta)}(P \cap Q)=A_{(\alpha, \beta)}(P) \cap A_{(\alpha, \beta)}(Q)$,

(5) $A_{(\alpha, \beta)}(P \bigcup Q) \supseteq A_{(\alpha, \beta)}(P) \bigcup A_{(\alpha, \beta)}(Q)$ equality holds if $\alpha+\beta=1$,

(6) $A_{(\alpha, \beta)}\left(\bigcap P_{i}\right)=\bigcap A_{(\alpha, \beta)}\left(P_{i}\right)$,

(7) $A_{(1,0)}(P)=X$.

Proof. (1) Let $x \in A_{(\alpha, \beta)}(P) \Rightarrow t_{p}(x) \geq \alpha$ and $f_{p}(x) \leq \beta$. Since $\gamma \leq \alpha$ and $\delta \geq \beta, \alpha \geq \gamma$ and $\beta \leq \delta: \Rightarrow t_{p}(x) \geq \alpha \geq \gamma$ and $f_{p}(x) \leq \beta \leq \delta$ $\Rightarrow t_{p}(x) \geq \gamma$ and $f_{p}(x) \leq \delta$

$\Rightarrow x \in A_{(\gamma, \delta)}$ therefore Let $x \in A_{(\alpha, \beta)}(P) \Rightarrow x \in A_{(\gamma, \delta)}$

$\Rightarrow A_{(\alpha, \beta)}(P) \subseteq A_{(\gamma, \delta)}(Q)$.

(2) Since $\alpha+\beta \leq 1 \Rightarrow \alpha \leq 1-\beta$ and $\beta \leq 1-\alpha$. Therefore using part (1), we obtain

$$
A_{(1-\beta, \beta)}(P) \subseteq A_{(\alpha, \beta)}(P) .
$$

Again $\alpha+\beta \leq 1 \Rightarrow \alpha \geq \alpha$ and $\beta \leq 1-\alpha$. Therefore using part (1) we obtain

$$
A_{(\alpha, \beta)}(P) \subseteq A_{(\alpha, 1-\alpha)}(P)
$$

From (1) and (2) we get $A_{(1-\beta, \beta)}(P) \subseteq A_{(\alpha, \beta)}(P) \subseteq A_{(\alpha, 1-\alpha)}(P)$.

(3) Let $x \in A_{(\alpha, \beta)}(P) \Rightarrow t_{p}(x) \geq \alpha$ and $f_{p}(x) \leq \beta$, as $Q \supseteq P \Rightarrow t_{Q}(x) \geq$ $t_{P}(x) \geq \alpha$ and $f_{p}(x) \leq \beta \leq \beta, \Rightarrow t_{P}(x) \geq \alpha$ and $f_{p}(x) \leq \beta \Rightarrow x \in A_{(\alpha, \beta)}(Q)$, $\Rightarrow x \in A_{(\alpha, \beta)}(P) \Rightarrow x \in A_{(\alpha, \beta)}(Q)$.

Therefore $A_{(\alpha, \beta)}(P) \subseteq A_{(\alpha, \beta)}(Q)$.

(4) $(P \cap Q) \subseteq P$ and $(P \cap Q) \subseteq Q$. Therefore by $(1)$ :

$$
A_{(\alpha, \beta)}(P \bigcap Q) \subseteq A_{(\alpha, \beta)}(P) \text { and } A_{(\alpha, \beta)}(P \bigcap Q) \subseteq A_{(\alpha, \beta)}(Q)
$$

Also, let $x \in X A_{(\alpha, \beta)}(P) \bigcap A_{(\alpha, \beta)}(Q), x \in X A_{(\alpha, \beta)}(P)$ and $x \in X A_{(\alpha, \beta)}(Q)$ $\Rightarrow t_{P}(x) \geq \alpha$ and $f_{p}(x) \leq \beta$ and $t_{Q}(x) \geq \alpha$ and $f_{Q}(x) \leq \beta$ 
$\Rightarrow t_{P}(x) \geq \alpha$ and $t_{Q}(x) \geq \alpha ; f_{P}(x) \leq \beta$ and $f_{Q}(x) \leq \beta$

$\Rightarrow t_{P}(x) \geq \alpha$ and $t_{Q}(x) \geq \alpha ; f_{P}(x) \leq \beta$ and $f_{Q}(x) \leq \beta$

$\Rightarrow t_{P}(x) \wedge t_{Q}(x) \geq \alpha ; f_{P}(x) \wedge f_{Q}(x) \leq \beta$

$\Rightarrow\left(t_{P} \wedge t_{Q}\right)(x) \geq \alpha ;\left(f_{P} \wedge f_{Q}\right)(x) \leq \beta$

$x \in A_{(\alpha, \beta)}(P \cap Q)$.

Thus

$$
A_{(\alpha, \beta)}(P) \bigcap A_{(\alpha, \beta)}(Q) \subseteq A_{(\alpha, \beta)}(P \bigcap Q) .
$$

From (3) and (4), we get $A_{(\alpha, \beta)}(P \cap Q)=A_{(\alpha, \beta)}(P) \cap A_{(\alpha, \beta)}(Q)$.

(5) Since $P \subseteq(P \bigcup Q)$ and $Q \subseteq(P \bigcup Q)$. Therefore using part (1):

$$
\begin{gathered}
A_{(\alpha, \beta)}(P) \subseteq A_{(\alpha, \beta)}(P \bigcup Q) \text { and } A_{(\alpha, \beta)}(Q) \subseteq A_{(\alpha, \beta)}(P \bigcup Q), \\
\Rightarrow A_{(\alpha, \beta)}(P) \bigcup A_{(\alpha, \beta)}(Q) \subseteq A_{(\alpha, \beta)}(P \bigcup Q)
\end{gathered}
$$

Now equality holds if $\alpha+\beta=1$ we show that

$$
A_{(\alpha, \beta)}(P \bigcup Q) \subseteq A_{(\alpha, \beta)}(P) \bigcup A_{(\alpha, \beta)}(Q) .
$$

Let $x \in A_{(\alpha, \beta)}(P \bigcup Q)$

$\Rightarrow\left(t_{p} \bigcup t_{q}(x)\right) \geq \alpha$ and $\left(f_{p} \bigcup f_{q}(x)\right) \leq \beta$

$\Rightarrow\left(t_{P}(x) \bigvee t_{Q}(x) \geq \alpha\right.$ and $f_{P}(x) \wedge f_{Q}(x) \leq \beta$.

If $t_{P}(x) \geq \alpha$, then $f_{P}(x) \leq 1-t_{P}(x) \leq 1-\alpha=\beta$ implies that $x \in$ $A_{(\alpha, \beta)}(Q) \subseteq A_{(\alpha, \beta)}(P) \bigcup A_{(\alpha, \beta)}(Q)$.

Also

$$
A_{(\alpha, \beta)}(P \bigcup Q) \subseteq A_{(\alpha, \beta)}(P) \cup A_{(\alpha, \beta)}(Q) .
$$

From (5) and (6), we get $A_{(\alpha, \beta)}(P \bigcup Q) \subseteq A_{(\alpha, \beta)}(P) \cup A_{(\alpha, \beta)}(Q)$.

(6) let $x \in A_{(\alpha, \beta)}\left(\bigcap P_{i}\right) \Rightarrow t_{p_{i}}(x) \geq \alpha$ and $\bigcap f_{P_{i}}(x) \leq \beta$ and $\bigwedge t_{P_{i}}(x) \geq \alpha$ and $\bigcap f_{p_{i}} \leq \beta \Rightarrow x \in A_{(\alpha, \beta)} P_{i}$ for all $\mathrm{i} \Rightarrow x \in A_{(\alpha, \beta)} \cap\left(P_{i}\right) \Rightarrow$ $x \in A_{(\alpha, \beta)}\left(P_{i}\right)$ for all $\mathrm{i}$, and hence

$$
A_{(\alpha, \beta)}\left(\bigcap P_{i}\right)=\bigcap A_{(\alpha, \beta)}\left(P_{i}\right) .
$$

(7) Since $A_{(\alpha, \beta)}(P)=\left\{X: x \in X: t_{P}(x) \geq \alpha\right.$ and $\left.f_{P}(x) \leq \beta\right\}$.

Setting $\alpha=0$ and $\beta=1, \Rightarrow A_{(\alpha, \beta)}(P)=X$.

Theorem 2.6. If $P$ is vague group of $G$. Then

$$
T_{\theta+}\left(A_{(\alpha, \beta)}(P)\right) \text { and } T_{\theta-}\left(A_{(\alpha, \beta)}(P)\right)
$$


are a translate vague group of $G$, where $t_{P}(e) \geq \alpha, f_{P}(e) \leq \beta$ and $e$ is the identity element of $G$.

Proof. Let $P$ is vague group of $G . A_{(\alpha, \beta)}(P)$ is vague cut-set. We shall show that $T_{\theta+}\left(A_{(\alpha, \beta)}(P)\right)$ is a translate of vague group of $G$.

We have:

$$
\begin{aligned}
t_{A(\alpha, \beta)_{T \theta+}}\left(x y^{-1}\right) & \geq \min \left\{t_{A_{(\alpha, \beta)}}\left(x y^{-1}\right)+\theta, 1\right\} \\
& \left.\geq \min \left\{t_{A_{(\alpha, \beta)}}(x)+\theta, 1\right\} \bigwedge\left\{t_{A_{(\alpha, \beta)}}\left(y^{-1}\right)+\theta, 1\right\}\right\} \\
& \left.=\min \left\{t_{A_{(\alpha, \beta)}}(x)+\theta, 1\right\},\left\{t_{A_{(\alpha, \beta)}}(y)+\theta, 1\right\}\right\} \\
& =\min \left\{t_{A_{(\alpha, \beta)}}(x),\left\{t_{A_{(\alpha, \beta)}}(y)\right\} .\right.
\end{aligned}
$$

Therefore

$$
\begin{gathered}
t_{A(\alpha, \beta)_{T \theta+}}\left(x y^{-1}\right) \geq \min \left\{t_{A_{(\alpha, \beta)}}(x), t_{A_{(\alpha, \beta)}}(y)\right\} \\
f_{A}(\alpha, \beta) T_{\theta+}\left(x y^{-1}\right) \leq \max \left\{f_{A_{(\alpha, \beta)}}\left(x y^{-1}\right)+\theta, 0\right\} \\
\left.\max \left\{f_{A_{(\alpha, \beta)}}\left(x y^{-1}\right)+\theta, 0\right\} \bigvee f_{A_{(\alpha, \beta)}}\left(y^{-1}\right)+\theta, 0\right\} \\
\max \left\{f_{A_{(\alpha, \beta)}}(x) \bigvee f_{A_{(\alpha, \beta)}}(y)\right\} .
\end{gathered}
$$

Therefore

$$
f_{A}(\alpha, \beta) T_{\theta+}\left(x y^{-1}\right) \leq \max \left\{f_{A_{(\alpha, \beta)}}(x), f_{A_{(\alpha, \beta)}}(y)\right\} .
$$

Hence from (1) and (2) it follows that $T_{\theta+}\left(A_{(\alpha, \beta)}(P)\right)$ is a vague group of $G$.

Similarly, we can show that $T_{\theta-}\left(A_{(\alpha, \beta)}(P)\right)$ is a vague group of $G$.

Theorem 2.7. $P$ and $Q$ are two translates of vague groups of $G$ then $P \cap Q$ is translates of vague group of $G$.

Proof. $P$ and $Q$ are two translates of vague groups of $G$. Then $T_{\theta+(P \cap Q)}$ is a translates of vague group of $\mathrm{G}$. Since the result hods true.

Now

$$
\begin{aligned}
t_{(P \cap Q) T_{\alpha+}}(x y) & \geq \min \left\{t_{(P \cap Q)}(x y)+\alpha, 1\right\}, \\
\left.\min \left\{t_{(P \cap Q)}(x)+\alpha, 1\right\},\left\{t_{(P \cap Q)}(y)+\alpha, 1\right\}\right\} & =\min \left\{t_{(P \cap Q)}(x), t_{(P \cap Q)}(y)\right\},
\end{aligned}
$$

Therefore

$$
t_{(P \cap Q) T_{\alpha+}}\left(x y^{-1}\right) \geq \min \left\{t_{(P \cap Q)}(x), t_{(P \cap Q)}(y)\right\}
$$




$$
\begin{aligned}
f_{(P \cap Q) T_{\alpha+}}\left(x y^{-1}\right) \leq \max & \left\{f_{(P \cap Q)}\left(x y^{-1}\right)-\alpha, 0\right\} \\
& \left.=\max \left\{f_{(P \cap Q)}(x)-\alpha, 0\right\},\left\{f_{(P \cap Q)}\left(y^{-1}\right)-\alpha, 0\right\}\right\}
\end{aligned}
$$

$\left.\max \left\{f_{(P \cap Q)}(x)-\alpha, 0\right\},\left\{f_{(P \cap Q)}(y)-\alpha, 0\right\}\right\}=\max \left\{f_{(P \cap Q)}(x), f_{(P \cap Q)}(y)\right\}$.

Hence

$$
f_{(P \cap Q) T_{\alpha+}}\left(x y^{-1}\right) \geq \max \left\{f_{(P \cap Q)}(x), f_{(P \cap Q)}(y)\right\} .
$$

From (1) and (2): $T_{\theta+(P \cap Q)}$ is a translates of vague group of $G$.

Theorem 2.8. Let $A$ be a translates of vague group of $G$. Then $\alpha$-cut $A_{\alpha}$ is a translates of vague group of $G$.

Proof. Let $A_{\alpha}=\left\{x \in G: t_{A}(x) \geq \alpha\right\}$ and let $x, y \in A_{\alpha}$

$$
\begin{gathered}
\Rightarrow t_{A T_{\alpha+}}\left(x y^{-1}\right)=\min \left\{t_{A}\left(x y^{-1}\right)+\alpha, 1\right\} \geq \min \left\{t_{A}(x)+\alpha, 1 t_{A}\left(y^{-1}\right)+\alpha, 1\right\} \\
=\min \left\{t_{A}(x), t_{A}(y)\right\}=\{\alpha, \alpha\} \\
\Rightarrow t_{A T_{\alpha+}}\left(x y^{-1}\right) \geq \min \{\alpha, \alpha\}=\alpha .
\end{gathered}
$$

So $x y^{-1} \in G$. Therefore

$$
\begin{gathered}
t_{A T_{\alpha+}}\left(x y^{-1}\right) \geq \min \left\{t_{A}(x), t_{A}(y)\right\} . \\
f_{A T_{\alpha+}}\left(x y^{-1}\right)=\max \left\{f_{A}\left(x y^{-1}\right)-\alpha, 0\right\} \leq \max \left\{f_{A}(x)-\alpha, 0 f_{A}\left(y^{-1}\right)-\alpha, 0\right\} \\
=\max \left\{f_{A}(x), f_{A}(y)\right\}=\{\alpha, \alpha\}, \\
\Rightarrow f_{A T_{\alpha+}}\left(x y^{-1}\right) \leq \max \{\alpha, \alpha\}=\alpha .
\end{gathered}
$$

So $x y^{-1} \in G$,

$$
f_{A T_{\alpha+}}\left(x y^{-1}\right) \leq \max \left\{f_{A}(x), f_{A}(y)\right\} .
$$

From (1) and (2) $A_{\alpha}$ cut set is a translates of vague group of $G$. 


\section{Translate Operators on Vague Homomorphism Groups and Vague Normal Groups}

Defination 3.1. Let $G$ be a vague normal group of be a vague set $\mathrm{P}$ then the translate operators of Vague normal group can be defined by

$T_{\alpha+}\left(V_{P}(x y)\right)=T_{\alpha+}\left(V_{P}(y x) \Rightarrow\left(t_{P \alpha+}(x y), f_{P \alpha+}(x y)\right)=\left(t_{P \alpha+}(y x), f_{P \alpha+}(y x)\right)\right.$

and

$T_{\alpha-}\left(V_{P}(x y)\right)=T_{\alpha-}\left(V_{P}(y x) \Rightarrow\left(t_{P \alpha-}(x y), f_{P \alpha-}(x y)\right)=\left(t_{P \alpha-}(y x), f_{P \alpha-}(y x)\right)\right.$.

Theorem 3.2. If $P$ is a translate of vague normal group of G. Then $T_{\theta+}\left(A_{(\alpha, \beta)}(P)\right)$ is a translate of vague normal group of $G$. where $t_{P}(e) \geq \alpha, f_{P}(e) \leq \beta$ and $e$ is the identity element of $G$.

Proof. Given that $\mathrm{P}$ is a vague normal group of $\mathrm{G}$ ie $V_{P}(x y)=V_{P}(y x)$ forall $\in \operatorname{Giet}_{P}(x y)=t_{P}(y x)$ and $f_{P}(x y)=f_{P}(y x)$ for all $x, y \in G$.

Now we shall that $T_{\theta+}\left(A_{(\alpha, \beta)}(P)\right)$ is Translate of vague normal group of $\mathrm{G}$, i.e.

$$
T_{\theta+}\left(A_{(\alpha, \beta)}(x y)\right)=T_{\theta+}\left(A_{(\alpha, \beta)}(y x)\right)=\left(t_{A_{(\alpha, \beta) T_{\theta+}}}, f_{A_{(\alpha, \beta) T_{\theta+}}}\right) .
$$

Now

$$
\begin{aligned}
& t_{A_{(\alpha, \beta) T_{\theta+}}}(x y) \geq \min \left\{t_{A_{(\alpha, \beta)}}(x y)+\theta, 1\right\}=\min \left\{t_{A_{(\alpha, \beta)}}(y x)+\theta, 1\right\} \\
& \Rightarrow t_{A_{(\alpha, \beta) T_{\theta+}}}(x y)=t_{A_{(\alpha, \beta) T_{\theta+}}}(y x), \\
& f_{A_{(\alpha, \beta) T_{\theta+}}(x y) \leq \max \left\{f_{A_{(\alpha, \beta)}}(x y)-\theta, 0\right\}=} \min \left\{f_{A_{(\alpha, \beta)}}(y x)-\theta, 0\right\} \\
& f_{A_{(\alpha, \beta) T_{\theta+}}(x y)}=f_{A_{(\alpha, \beta)}}(y x) .
\end{aligned}
$$

From (1) and (2):

$$
T_{\theta+}\left(A_{(\alpha, \beta)}(x y)\right)=T_{\theta+}\left(A_{(\alpha, \beta)}(y x)\right) .
$$

Therefore $T_{\theta+}\left(A_{(\alpha, \beta)}(P)\right)$ is translate of vague normal group of $G$.

Theorem 3.3. Let $G$ and $G^{1}$ be any two vague groups then the homomorphic image of translates of vague group $A$ of $G$ is a vague group of $G^{1}$. 
Proof. Let $\mathrm{G}$ and $G^{1}$ be any two groups and $\Phi: G \rightarrow G^{1}$ be a homomorphism.

Therefore $\Phi(x, y)=\Phi(x) . \Phi(y)$ for all $x, y \in G$. Let $V=\Phi\left(T_{\alpha+}\right)$ be a translates of a vague group of $\mathrm{G}$. We shall show that $V=\Phi\left(T_{\alpha+}\right)$ is a vague group of $G^{1}$.

For $\Phi(x), \Phi(y) i n G^{1}$, we have

$$
\begin{array}{r}
t_{P T_{\alpha+}}\left(\Phi(x) \Phi\left(y^{-1}\right)\right) \leq \min \left\{t_{P} \Phi\left(x y^{-1}\right)+\alpha, 1\right\}=\min \left\{t_{P}\left\{\Phi(x)\left(y^{-1}\right)+\alpha, 1\right\}\right. \\
=\min \left\{\left\{t_{P} \Phi(x)+\alpha, 1\right\},\left\{t_{P} \Phi(y)+\alpha, 1\right\}\right\} .
\end{array}
$$

Therefore

$$
\begin{gathered}
t_{P T_{\alpha+}}\left(\Phi(x) \Phi\left(y^{-1}\right)\right) \leq \min \left\{t_{P} \Phi(x), t_{P} \Phi(y)\right\} . \\
f_{P T_{\alpha+}}\left(\Phi(x) \Phi\left(y^{-1}\right)\right) \leq \max \left\{t_{P} \Phi\left(x y^{-1}\right)-\alpha, 0\right\}=\max \left\{f_{P}\left\{\Phi(x)\left(y^{-1}\right)-\alpha, 0\right\}\right. \\
=\max \left\{\left\{t_{P} \Phi(x)-\alpha, 0\right\},\left\{t_{P} \Phi(y)-\alpha, 0\right\}\right\} .
\end{gathered}
$$

Therefore

$$
f_{P T_{\alpha+}}\left(\Phi(x) \Phi\left(y^{-1}\right)\right) \leq \max \left\{f_{P} \Phi(x), f_{P} \Phi(y)\right\} .
$$

From (1) and (2): $V=\Phi\left(T_{\alpha+}\right)$ is a translate of vague group of $G^{1}$.

Theorem 3.4. Let $G$ and $G^{1}$ be any two vague groups. Then the homomorphic image of an translates of vague normal group $A$ of $G$ is an vague normal group of $G^{1}$.

Proof. Let $\mathrm{G}$ and $G^{1}$ be any two groups and $\Phi: G \rightarrow G^{1}$ be a homomorphism. $G$.

Let $V=\Phi\left(T_{\alpha+}\right)$ where $T_{\alpha+}$ is a translation of vague normal group of $A$ of

We shall show that $V=\Phi\left(T_{\alpha+}\right)$ is a translates of vague normal group $G^{1}$.

For any $\Phi(x), \Phi(y) i n G^{1}$, we have, for any $\Phi(x), \Phi(y)$ in $G^{1}$,

$$
T_{\alpha+} \Phi(x y)=\left(t_{P} T_{\alpha+} \Phi(x y), f_{P} T_{\alpha+} \Phi(x y)\right) .
$$

Consider

$$
\begin{gathered}
t_{P} T \alpha+\Phi(x y)=\min \left\{t_{P T_{\alpha+}} \Phi(x y)+\alpha, 1\right\}=\min \left\{t_{P T_{\alpha+}} \Phi(y x)+\alpha, 1\right\} \\
\Rightarrow t_{P} T \alpha+\Phi(x y)=t_{P T_{\alpha+}}\left(\Phi(y x) T_{\alpha+} \Phi(x y)=\left(t_{P} T_{\alpha+} \Phi(x y), f_{P} T_{\alpha+} \Phi(x y)\right) .\right.
\end{gathered}
$$


Moreover

$$
f_{P} T \alpha+\Phi(x y)=\max \left\{f_{P T_{\alpha+}} \Phi(x y)-\alpha, 0\right\}=\max \left\{f_{P T_{\alpha+}} \Phi(y x)-\alpha, 0\right\}
$$

Hence $f_{P} T \alpha+\Phi(x y)=f_{P T_{\alpha+}}(\Phi(y x)$.

Theorem 3.5. Let $G$ and $G^{1}$ be any two vague groups . Then the homomorphic pre-image of an translates of vague group $V$ of $G^{1}$ is an vague group of G.

Proof. Let $\mathrm{G}$ and $G^{1}$ be any two groups and $\Phi: G \rightarrow G^{1}$ be homomorphism.

Let $V=\Phi\left(T_{\alpha+}\right)$ be translates of vague group of $G^{1}$. We shall show that $\mathrm{P}$ is a vague group $\mathrm{V}$ of $\mathrm{G}$.

Let $x, y \in G$. Then

$$
\begin{aligned}
& V=\Phi^{-1}\left(T_{\alpha+}\right)=\left(t_{A T_{\alpha+}} \phi^{-1}(x), f_{A T_{\alpha+}} \phi^{-1}(x)\right) \\
& t_{A T_{\alpha+}} \Phi^{-1}\left(x y^{-1}\right)=t_{A T_{\alpha+}} \Phi^{-1}\left(x y^{-1}\right)=t_{A T_{\alpha+}}\left(\Phi^{-1}(x) \Phi^{-1}\left(y^{-1}\right)\right) \\
& \geq \min \left\{t_{P}\left[\left(\Phi^{-1}(x) \Phi^{-1}\left(y^{-1}\right)\right)\right]+\alpha, 1\right\}=\min \left\{\left(t_{P}\left(\Phi^{-1}(x)+\alpha\right) \bigwedge\left(\Phi^{-1}\left(y^{-1}\right)\right)+\alpha, 1\right)\right\} \\
& \min \left\{\left(t_{P}\left(\Phi^{-1}(x)+\alpha\right) \bigwedge\left(\Phi^{-1}\left(y^{-1}\right)\right)+\alpha, 1\right)\right\} \\
& =\min \left\{\left\{t_{P} \Phi^{-1}(x)+\alpha, 1\right\},\left\{t_{P} \Phi^{-1}(y)+\alpha, 1\right\}\right\} \\
& \min \left\{t_{P} \Phi^{-1}(x) \bigwedge t_{P} \Phi^{-1}(y)\right\}=\min \left\{t_{P}(x) t_{P}(y)\right\}
\end{aligned}
$$

Therefore

$$
\begin{gathered}
t_{A T_{\alpha+}} \Phi^{-1}\left(x y^{-1}\right) \geq \min \left\{t_{P}(x) t_{P}(y)\right\} \\
f_{A T_{\alpha+}} \Phi^{-1}\left(x y^{-1}\right)=f_{A T_{\alpha+}} \Phi^{-1}\left(x y^{-1}\right)=f_{A T_{\alpha+}}\left(\Phi^{-1}(x) \Phi^{-1}\left(y^{-1}\right)\right) \\
\leq \max \left\{f_{P}\left[\left(\Phi^{-1}(x) \Phi\left(y^{-1}\right)\right)\right]-\alpha, 0\right\}=\max \left\{\left(f_{P}\left(\Phi^{-1}(x)-\alpha\right) \bigwedge\left(\Phi\left(y^{-1}\right)\right)-\alpha, 0\right)\right\} \\
\max \left\{\left(f_{P}\left(\Phi^{-1}(x)-\alpha\right) \bigwedge\left(\Phi^{-1}\left(y^{-1}\right)\right)-\alpha, 0\right)\right\}=\max \left\{\left\{f_{P} \Phi^{-1}(x)-\alpha, 0\right\}\right. \\
\left.\left\{f_{P} \Phi^{-1}(y)-\alpha, 0\right\}\right\}=\max \left\{f_{P} \Phi^{-1}(x) \bigwedge f_{P} \Phi^{-1}(y)\right\}=\max \left\{f_{P}(x), f_{P}(y)\right\} .
\end{gathered}
$$

Hence

$$
f_{A T_{\alpha+}} \Phi^{-1}\left(x y^{-1}\right) \geq \max \left\{f_{P}(x), f_{P}(y)\right\} .
$$

From (1) and (2) the homomorphic pre-image of an translates of vague group $\mathrm{V}$ of $G^{1}$ is an vague group of $\mathrm{G}$. 
Theorem 3.6. Let $G$ and $G^{1}$ be any two vague groups . Then the homomorphic pre-image of an translates of vague group of a vague normal group $V$ of $G^{1}$ is translates of vague normal group of $G$.

Proof. Let $\mathrm{G}$ and $G^{1}$ be any two groups and $\Phi: G \rightarrow G^{1}$ be a homomorphism.

Let $V=\Phi^{-1}\left(T_{\alpha+}\right)$ where $T_{\alpha+}$ is a translates of vague operators of vague normal group of $\mathrm{G}$. We shall show that $\mathrm{P}$ is vague normal group of $\mathrm{G}$. Now, Let $x, y \in G \Rightarrow V=\Phi^{-1}\left(T_{\alpha+}\right)=\left(t_{P T_{\alpha+}} \phi^{-1}(x), f_{P T_{\alpha+}} \phi^{-1}(x)\right)$.

Consider

$$
\begin{array}{r}
t_{P T_{\alpha+}} \phi^{-1}(x y) \geq \min \left\{t_{P} \phi^{-1}(x y)+\alpha, 1\right\}=\min \left\{t_{P} \phi^{-1}(y x)+\alpha, 1\right\} \\
=t_{P T_{\alpha+}} \phi^{-1}(y x) t_{P T_{\alpha+}} \phi^{-1}(x y)=t_{P T_{\alpha+}} \phi^{-1}(y x)
\end{array}
$$

for all $x, y \in G$. Also

$$
\begin{aligned}
f_{P T_{\alpha+}} \phi^{-1}(x y) \leq \max \left\{f_{P}^{-1}(x y)-\alpha, 0\right\}= & \max \left\{f_{P}^{-1}(y x)-\alpha, 0\right\}=f_{P T_{\alpha+}} \phi^{-1}(y x) \\
& f_{P T_{\alpha+}} \phi^{-1}(x y)=f_{P T_{\alpha+}} \phi^{-1}(y x), \quad(2)
\end{aligned}
$$

for all $x, y \in G$.

Therefore from (1)and(2) the homomorphic pre-image of an translates of vague group of a vague normal group $V$ of $G^{1}$ is translates of vague normal group of $\mathrm{G}$.

Theorem 3.7. If $P$ is translate of vague normal group of $G$, then $K=$ $\left\{x \in G: G V_{P}=G V_{P}(e)\right\}$ is translate of crisp normal group of $G$.

Proof. Let $\mathrm{P}$ is a translates of vague normal group of $\mathrm{G}$, let $\mathrm{x}, \mathrm{y} \in K \Rightarrow$ $V_{P}(x)=V_{P}(e)$ and $V_{P}(y)=V_{P}(e)$. Now

$$
\begin{aligned}
& t_{V_{P} T_{\alpha+}}\left(x y^{-1}\right) \geq \min \left\{t_{V_{P}}\left(x y^{-1}\right)+\alpha, 1\right\} \\
&= \min \left\{\left\{t_{V_{P}}(x)+\alpha, 1\right\},\left\{t_{V_{P}}\left(y^{-1}\right)+\alpha, 1\right\}\right\}=\min \left\{\left\{t_{V_{P}}(e)+\alpha, 1\right\},\left\{t_{V_{P}}(e)+\alpha, 1\right\}\right\} \\
& t_{V_{P} T_{\alpha+}}\left(x y^{-1}\right) \geq t_{V_{P}}(e) . \\
& f_{V_{P} T_{\alpha+}}\left(x y^{-1}\right) \leq \max \left\{f_{V_{P}}\left(x y^{-1}\right)-\alpha, 0\right\} \\
&=\max \left\{\left\{f_{V_{P}}(x)-\alpha, 0\right\},\left\{f_{V_{P}}\left(y^{-1}\right)-\alpha, 0\right\}\right\}=\max \left\{\left\{f_{V_{P}}(e)-\alpha, 0\right\},\left\{f_{V_{P}}(e)-\alpha, 0\right\}\right\} .
\end{aligned}
$$

Therefore

$$
f_{V_{P} T_{\alpha+}}\left(x y^{-1}\right) \leq f_{V_{P}}(e) .
$$


Hence from (1)and $(2) V_{P T_{\alpha+}}$ is a vague crisp group of $\mathrm{G}$.

Let $x \in G, y \in K$.Then

$$
t_{P T_{\alpha+}}(x y) \geq \min \left\{t_{P}(x y)+\alpha, 1\right\}=\min \left\{t_{P}(y x)+\alpha, 1\right\} .
$$

Therefore

$$
t_{P T_{\alpha+}}(x y)=t_{P T_{\alpha+}}(y x) .
$$

Let $x \in G, y \in K$. Then

$$
f_{P T_{\alpha+}}(x y) \leq \max \left\{f_{P}(x y)-\alpha, 0\right\}=\max \left\{f_{P}(y x)-\alpha, 0\right\} .
$$

Therefore $f_{P T_{\alpha+}}(x y)=f_{P T_{\alpha+}}(y x)$.

Hence $\mathrm{k}$ is translates of crisp normal group of $\mathrm{G}$.

Theorem 3.8. Let $P$ and $Q$ be two translates vague normal group of a group $G$. Then $(P \cap Q)$ is translates of vague normal group of $G$.

Proof. Since $P$ and $Q$ are a translates vague normal group of a group $G$ and Now we shall so that $(P \cap Q)$ is a translates vague normal group of $G$

$$
\begin{aligned}
& t_{(P \cap Q) T_{\alpha+}}(x y) \geq \min \left\{t_{(P \cap Q)}(x y)+\alpha, 1\right\} \\
& =\min \left\{t_{(P \cap Q)}(y x)+\alpha, 1\right\}=t_{(P \cap Q) T_{\alpha+}}(y x), \\
& f_{(P \cap Q) T_{\alpha+}}(x y) \leq \max \left\{f_{(P \cap Q)}(x y)+\alpha, 1\right\} \\
& =\max \left\{f_{(P \cap Q)}(y x)+\alpha, 1\right\}=f_{(P \cap Q) T_{\alpha+}}(y x) .
\end{aligned}
$$

Therefore $(P \cap Q)$ is translates of vague normal group of $\mathrm{G}$.

Theorem 3.9. Let $P$ be a translates of vague group of $G$. Then $K=\{x \in$ $\left.G: t_{P}(x)=t_{P}(e) a n d f_{P}(x)=f_{P}(e)\right\}$ is a translates of vague normal group of $N(P)$.

Proof. Let $x \in K$ and $\left.y \in K \Rightarrow t_{P}(x)=t_{P}(e) a n d f_{P}(x)=f_{P}(e)\right\}$. consider

$$
\begin{gathered}
\left.\left.t_{(P \cap Q) T_{\alpha+}\left(x y x^{-1}\right) \geq \min \left\{\left\{t_{(P \cap Q)}\left(x x^{-1}\right)+\alpha, 1\right\}\right.} t_{(P \cap Q)}(y)+\alpha, 1\right\}\right\} \\
=\min \left\{\left\{t_{(P \cap Q)}(e)+\alpha, 1\right\}\right. \\
\left.\left.t_{(P \cap Q)}\left(x y x^{-1}\right)+\alpha, 1\right\}\right\}=t_{(P \cap Q)}(e)=t_{(P \cap Q)}\left(x y x^{-1}\right) .
\end{gathered}
$$


Therefore $t_{(P \cap Q) T_{\alpha+}}\left(x y x^{-1}\right)=N(P)$ and

$$
\begin{gathered}
f_{(P \cap Q) T_{\alpha+}}\left(x y x^{-1}\right) \leq \max \left\{\left\{f_{(P \cap Q)}\left(x x^{-1}\right)-\alpha, 0\right\}\right. \\
\left.\left.f_{(P \cap Q)}(y)-\alpha, 0\right\}\right\}=\max \left\{\left\{t_{(P \cap Q)}(e)-\alpha, 0\right\}\right. \\
\left.f_{(P \cap Q)}\left(x y x^{-1}\right)-\alpha, 0\right\}=f_{(P \cap Q)}(e)=f_{(P \cap Q)}\left(x y x^{-1}\right)
\end{gathered}
$$

Therefore $f_{(P \cap Q) T_{\alpha+}}\left(x y x^{-1}\right)=N(P), K=\left\{x \in G: t_{P}(x)=t_{P}(e)\right.$, and $\left.f_{P}(x)=f_{P}(e)\right\}$ is a translates of vague normal group of $N(P)$.

Theorem 3.10. Let $P$ be a translates vague group of a group $G$, and $K=\left\{x \in G \mid V_{P}(x)=V_{P}(e)\right\}$ is translates of vague normal group of $G$. Then $K \subseteq C(P)$.

Proof. Let $x \in K$. Then

$$
V_{P}(x)=V_{P}(e) \Rightarrow t_{P}(x)=t_{P}(e)
$$

and

$$
\begin{gathered}
f_{P}(x)=f_{P}(e) \text { for all } x, y \in G . \\
t_{P T_{\theta+}}[x, y]=\min \left\{t_{P}[x, y]+\theta, 1\right\}=\min \left\{t_{P}\left(x^{-1} y^{-1} x y\right)+\theta, 1\right\} \\
\geq \min \left\{\left\{t_{P}\left(x^{-1} x\right)+\theta, 1\right\},\right. \\
\left.\left.t_{P}\left(y y^{-1}\right)+\theta, 1\right\}\right\}=\min \left\{\left\{t_{P}(e)+\theta, 1\right\}\right. \\
\left.\left.t_{P}(e)+\theta, 1\right\}\right\}=\min \left\{t_{P}(e), t_{P}(e)\right\}=t_{P}(e) .
\end{gathered}
$$

Therefore

$$
t_{P T_{\theta+}}[x, y]=t_{P}(e)
$$

$$
\begin{gathered}
f_{P T_{\theta+}}[x, y]=\max \left\{f_{P}[x, y]-\theta, 0\right\}=\max \left\{f_{P}\left(x^{-1} y^{-1} x y\right)-\theta, 0\right\} \\
\leq \max \left\{\left\{f_{P}\left(x^{-1} x\right)-\theta, 0\right\},\right. \\
\left.\left.f_{P}\left(y y^{-1}\right)-\theta, 0\right\}\right\}=\max \left\{\left\{f_{P}(e)-\theta, 0\right\}\right. \\
\left.\left.f_{P}(e) \theta, 0\right\}\right\}=\max \left\{f_{P}(e), f_{P}(e)\right\}=f_{P}(e) .
\end{gathered}
$$

Therefore

$$
t_{P T_{\theta+}}[x, y]=t_{P}(e) .
$$

Hence from (1) and(2) it follows $K \subseteq C(P)$. 


\section{Acknowledgements}

The authors are grateful to Prof. K.L.N. Swamy for his valuable suggestions and discussions on this work.

\section{References}

[1] K.T. Atanassov, More on intuitionistic fuzzy sets, Fuzzy Sets and Systems, 33 (1989), 37-45.

[2] B. Nageswararao, N. Ramakrishana, T. Eswarlal, Translates of vague groups, International Journal of Pure and Applied Mathematical Sciences, 7, No. 2 (2014), 217-226.

[3] W.L. Gahu, D.J. Buehrer, Vague sets, IEEE Transactions on Systems, Man. and Cybernetics, 23 (1993), 610-614.

[4] A. Goguen, L-fuzzy sets, J. Math. Anal. Applic., 18 (1967), 145-174.

[5] N.P. Mukharjee, Fuzzy normal subgroups and fuzzy cosets, Information Sciences, 34 (1984), 225-239.

[6] N. Ramakrishana, B. Nageswararao, T. Eswarlal, Ch. Satyanarayana, Anti-homomorphisms in vague groups, IJMSEA, 6, No. 2 (2012), 449-459.

[7] N. Ramakrishana, Ch. Satyanarayana, B. Nageswararao, IJMSEA, 6, No.3 (2012), 387-397.

[8] N. Ramakrishna, T. Eswarlal, G.S.V. Saibaba, A characterization of cyclic groups in terms of L-fuzzy subgroups, Southeast Asian Bulletin of Mathematics, 33 (2009), 913-916.

[9] Ranjit Biswas, Vague groups, Int. Journal of Computational Cognition, 4, No. 2 (2006).

[10] N. Ramakrishana, T. Eswarlal, Boolean vague sets, Int. Journal of Computional Congnition, 5, No. 4 (2007), 50-53.

[11] N. Ramakrishna, A chatracterization of cyclic in terms of vague groups, Int. Journal of Computational Congnition, 6, No. 2 (2008), 17-20.

[12] N. Ramakrishna, Vague groups and vague weights, Int. Journal of Computational Congnition, 66, No. 1 (2009), 913-916. 
[13] A. Rosenfeld, Fuzzy groups, Jon. Maths. Anal. Appli., 35 (1971), 512-517.

[14] L.A. Zadeh, Fuzzy sets, Infor and Control, 8 (1965), 338-353. 
